\title{
A simple and cost-effective model for ventricular catheter placement training: technical note
}

\author{
Nathan Todnem, MS, MD, ${ }^{1}$ Khoi D. Nguyen, MD, ${ }^{1}$ Vamsi Reddy, BS, ${ }^{1}$ Dayton Grogan, BS, ${ }^{1}$ \\ Taylor Waitt, BA, ${ }^{1}$ and Cargill H. Alleyne Jr., MD² \\ 'Department of Neurosurgery, Augusta University Medical Center; and 2University Neuroscience Augusta Back, Augusta,
Georgia
}

OBJECTIVE External ventricular drain (EVD) placement is one of first cranial procedures neurosurgery residents are expected to perform independently. While proper training improves patient outcomes, there are few options for practicing EVD placement prior to placing the EVD in patients in a clinical setting. Proposed solutions to this include using cadaveric models and virtual simulations, but barriers exist with these as well in regard to authenticity. EVD simulators using virtual reality technologies are a promising new technique for training, but the cost of these devices poses a barrier to general/widespread accessibility among smaller programs or underserved hospitals. The authors desribe a novel, yet simple, and cost-effective technique (less than $\$ 5$ per mold) for developing a brain model constructed of homemade ballistics gelatin that can be used for teaching and practicing the placement of EVD.

METHODS A brain model is made with ballistics gelatin using an anatomically correct skull model as a mold. A 3Dprinted ventricular system model is used to create a mold of an anatomically correct ventricular system in the brain model. A group of medical students $(n=10)$ were given a basic presentation about EVD placement, including standard landmarks and placement techniques, and were also shown a demonstration of EVD placement on the brain model. They were then allowed to perform an EVD placement using the brain model. The students were surveyed on their experience with using the brain model, including usability and practicality of the model. Accuracy of EVD placement by each student was also assessed, with adequate position of catheter tip being in the ipsilateral frontal horn.

RESULTS The final product is fairly inexpensive and easy to make. It is soft enough to pass a catheter through, but it is also firm enough to maintain its shape, including a cavity representing the lateral ventricles. The dense gelatin holds the catheter in its final resting position, while the two halves are separated and inspected. All participants in the test group of medical students reported that the brain model was easy to use, helped them understand the steps and technique of EVD placement, and provided good feedback on the ideal position of ventricular catheters. All of the participants in the group had adequate positioning of their ventricular catheters after one attempt.

CONCLUSIONS The presented brain model is easy to replicate, inexpensive, anatomically accurate, and provides a medium for neurosurgeons to teach and practice ventricular catheter placement in a risk-free environment.

https://thejns.org/doi/abs/10.3171/2020.2.JNS19161

KEYWORDS ventricular catheter placement; brain model; training; gelatin; hydrocephalus; surgical technique

$\mathrm{E}$ XTERNAL ventricular drain (EVD) placement is one of first cranial procedures neurosurgery residents are expected to perform independently. It is a vital and lifesaving skill that all practicing neurosurgeons should be capable of performing accurately and efficiently. Ventricular drains allow constant monitoring of intracranial pressure (ICP) and diversion of CSF in critically ill patients, such as those with acute hydrocephalus, traumatic brain injury, or intracranial hemorrhage. ${ }^{9}$

When EVDs are suboptimally placed, there are conse- quences, such as nonfunctional drains, infection, hemorrhage, progression of hydrocephalus, and iatrogenic injury to brain structures. ${ }^{1-4}$ While proper training improves patient outcomes, there are few options for practicing EVD placement prior to actually placing an EVD in patients in a clinical setting. ${ }^{1}$ In addition to the obvious risk of having unpracticed professionals doing procedures on patients, intraoperative procedural training has also been shown to increase operation time and cost..$^{5,10}$ Proposed solutions to this include using cadaveric models and virtual simu-

ABBREVIATIONS EVD = external ventricular drain; ICP = intracranial pressure.

SUBMITTED January 19, 2019. ACCEPTED February 13, 2020.

INCLUDE WHEN CITING Published online May 1, 2020; DOI: 10.3171/2020.2.JNS19161. 


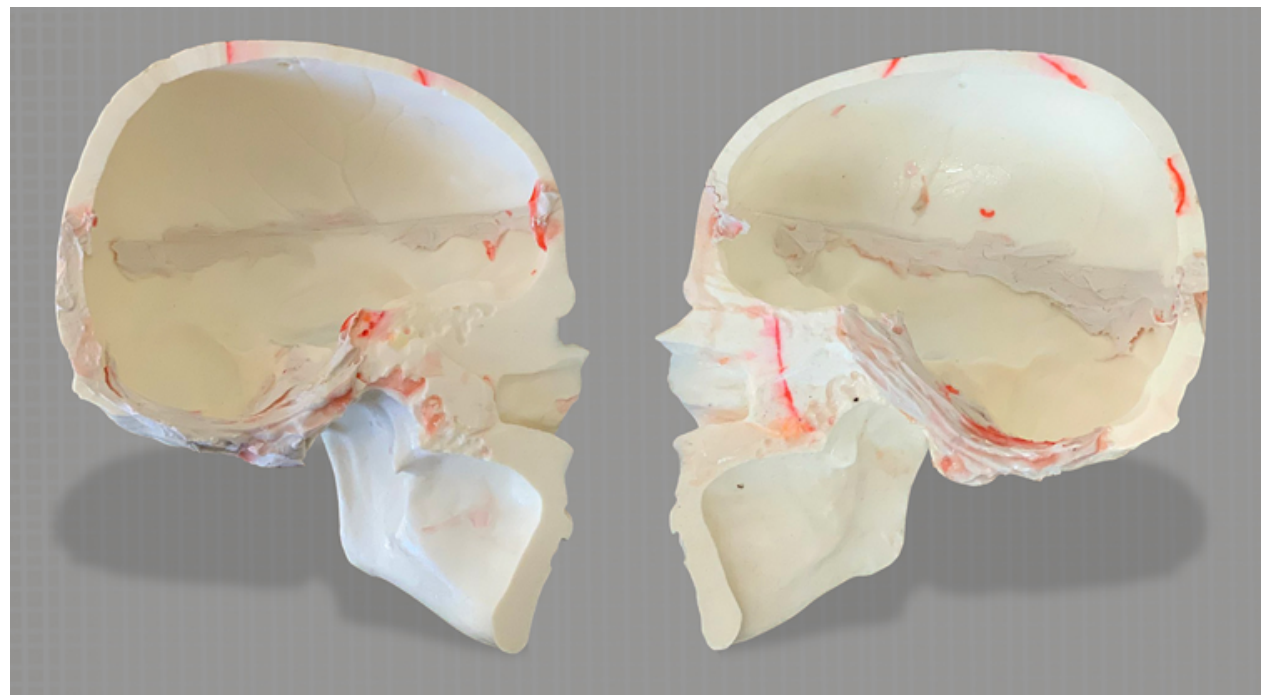

FIG. 1. Skull model used as mold to make gelatin brain model. Figure is available in color online only.

lations, but barriers exist with these as well in regard to authenticity. Morphological and structural tissue changes in cadavers make them poor parallels to living patients. EVD simulators using virtual reality technologies are a promising new technique for training, but they still fall short in simulating relevant aspects of the procedure such as lack of physical interaction and necessary steps taken before the placement of the EVD. ${ }^{6}$ Additionally, the cost of these devices poses a barrier to general/widespread accessibility among smaller programs or underserved hospitals. Our model is not only inexpensive (less than $\$ 5$ per mold), but each mold can be used 20-30 times, and the skull base can be reused indefinitely to make more molds.

We present a novel, yet simple, and cost-effective technique for developing a brain model constructed of homemade ballistics gelatin that can be used for teaching and practicing the placement of EVDs.

\section{Method}

\section{Brain Model Construction}

An anatomically correct skull model (hardened plastic)

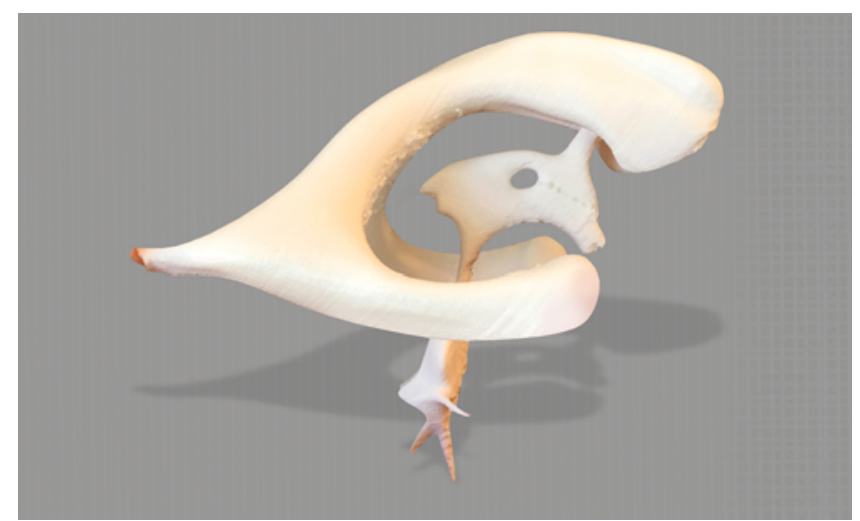

FIG. 2. 3D-printed model of the ventricular system. Figure is available in color online only. was separated in the midsagittal plane to create the base for the molds (Fig. 1). A 3D-printed ventricular model was generated and printed based on the average measurements from 10 consecutive normal MR images (Fig. 2). The 3D ventricular model was bisected in the midsagittal plane and secured to a piece of plastic to serve as the lid to the brain mold (Fig. 3). A recipe for homemade ballistics gelatin was followed; a solution of 1 ounce of gelatin powder for every 8 ounces of water is heated over medium heat until completely liquefied, and then it is refrigerated until solid. The solid is then reheated a second time to remove any trapped air and then poured into the bisected skull molds, and the appropriate halved ventricular molds are secured on top in correct anatomical position (Fig. 4). After cooling, the gelatin models can be removed from the molds and secured into an intact upright model skull to be used for teaching (Fig. 5).

\section{Testing of Brain Model}

Prior to testing the model, senior neurosurgeons and residents performed trials on the model to confirm that it is realistic. A group of medical students $(n=10)$ were given a basic presentation about EVD placement including standard landmarks and placement techniques, and

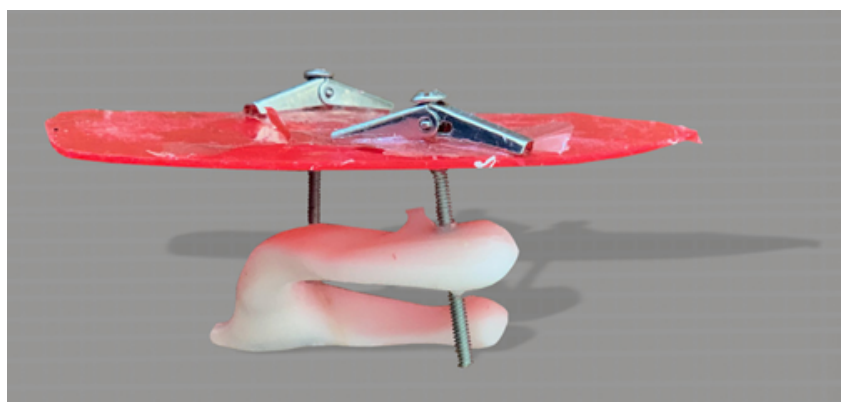

FIG. 3. Ventricular system model cut in half and secured to plastic plate. Figure is available in color online only. 


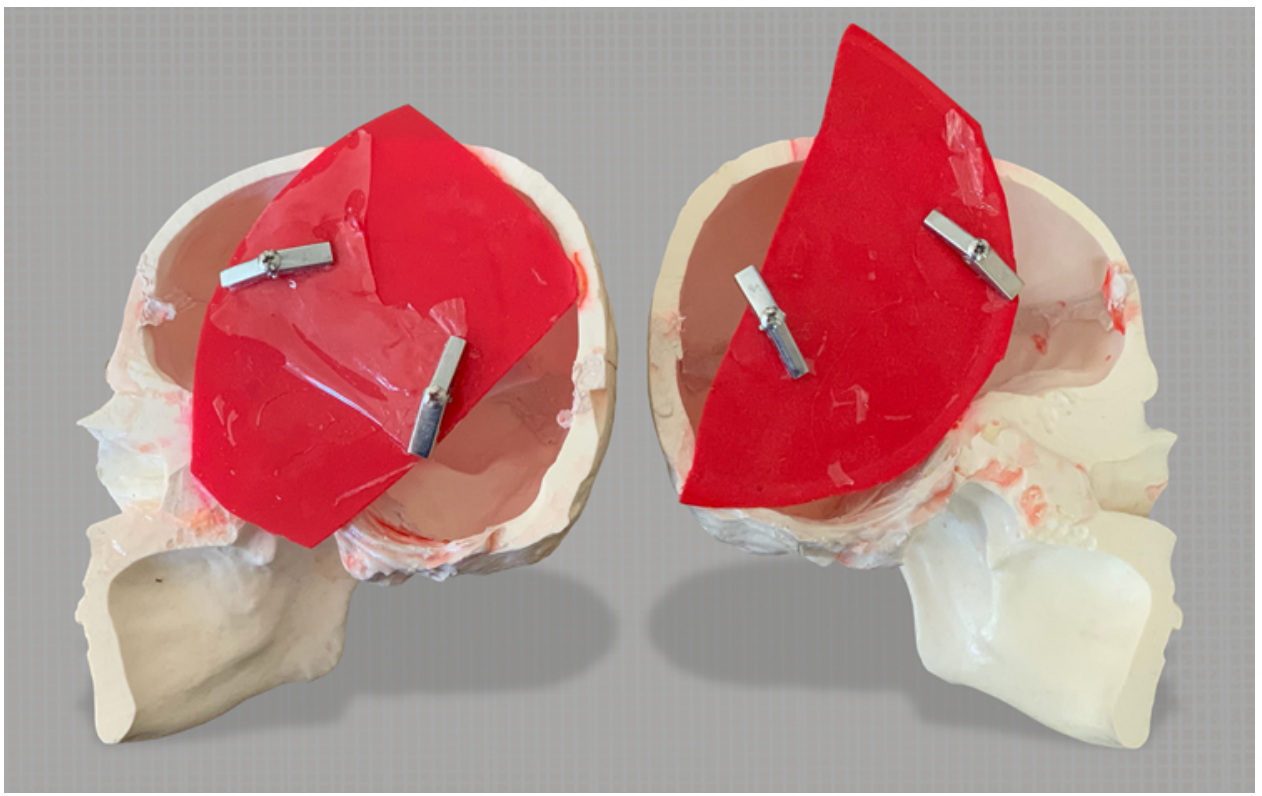

FIG. 4. Completed mold with lids in place, prior to adding in gelatin. The lids are placed in an orientation so that the ventricular models are in an anatomically correct position. Figure is available in color online only.

were also shown a demonstration of EVD placement on the brain model. This presentation lasted approximately 15 minutes. They were then allowed to perform an EVD placement using the brain model. The students were surveyed on their experience with using the brain model. This survey including ratings of usability and practicality of the model. The accuracy of EVD placement by each student was also assessed by residents, with adequate position of the catheter tip being in the ipsilateral frontal horn.

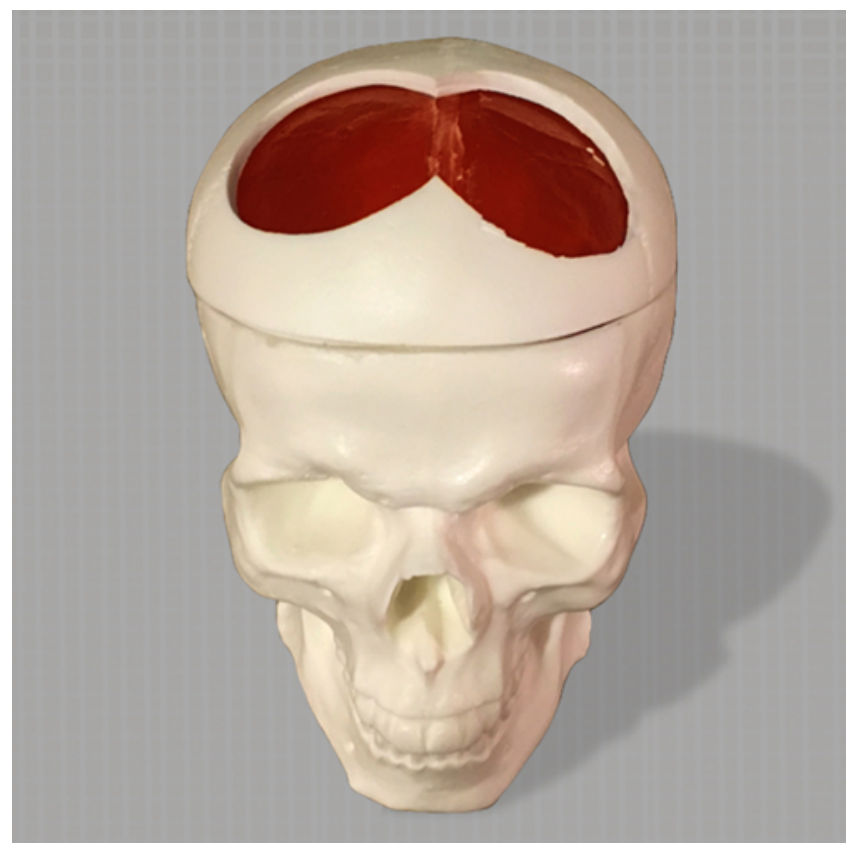

FIG. 5. Completed gelatin brain model in skull model. Figure is available in color online only.

\section{Results}

The final product is fairly inexpensive and easy to make. It is soft enough to pass a catheter through, but it is also firm enough to maintain its shape, including a cavity representing the lateral ventricles. The dense gelatin holds the catheter in its final resting position while the two halves are separated and inspected (Fig. 6). Ten out of 10 participants in the test group of medical students reported via individual interviews that the brain model was easy to use, helped them understand the steps and technique of EVD placement, and provided excellent feedback on the ideal position of ventricular catheters. All of the same 10 participants in the group had adequate positioning of their ventricular catheters after one attempt, as assessed by senior residents. This was able to be assessed easily due to the ability of the gelatin mold to be separated via brain hemispheres.

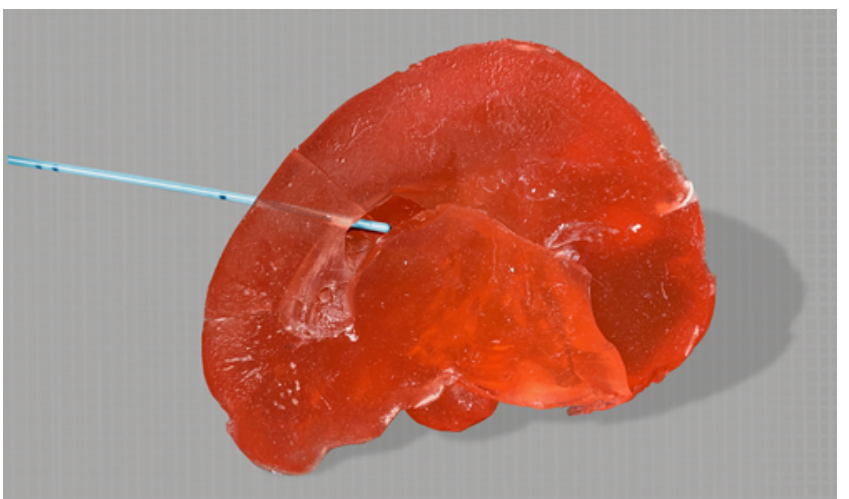

FIG. 6. A ventricular catheter is inserted into the gelatin brain model, with a view of the catheter tip in the ventricle. Figure is available in color online only. 


\section{Discussion}

Given the need for increased accuracy in correctly placing an EVD, it is essential that training models represent the anatomical structure and consistency of the human brain as closely as possible. ${ }^{11}$ Moreover, it is necessary that this training be active and performed using the same instruments as appear in the actual clinical setting. While current models attempt to satisfy these requirements, the use of hard plastics and cadaver brains is unrealistic because of their consistency and structure and because they are neither easily accessible nor reusable. ${ }^{7}$ The use of virtual reality models, at this time, also presents challenges of replicating anatomical accuracy and being easily accessible or inexpensive. ${ }^{4,6,8}$ The use of ballistics gelatin molds allows for the ability to readily generate anatomically accurate brain models that provide immediate feedback and are easily reusable. Alterations to the gelatin's recipe allow for the generation of various consistencies that may mimic neurological structure in numerous scenarios.

The use of such gelatin models not only offers a more realistic and available manner for training neurosurgeons in the placement of EVDs, but also demonstrates high degrees of achieved accuracy. ${ }^{12}$ There was a high rate of accurate ventricular catheter placement during testing of our brain model by medical students. Given these data, ballistics gelatin models may offer an affordable and reliable modality with which neurosurgery residents may train. Additional testing trials with a higher number of medical students and neurosurgical residents are the next step in further assessing the feasibility of our brain model. Eventually, as more trials are performed, this brain model will be compared to other ventriculostomy models, including plastic and cadaver models, as well as virtual realitybased models.

Limitations include a lower sample size as the authors did not intend for this to be a scientific study, but rather a proof of concept for the teachability and replicability of such a model. Furthermore, the gelatin mold can only be stored at room temperature for 24-48 hours before it starts to soften. This issue was combatted by storing the molds in the freezer and letting them thaw for 15 minutes prior to using them for practice. This model also only represents EVD placement after skin incision and burr hole creation, as we intend this to be a cost-effective model and skin-like material over the incision site could be costly and is not reusable.

\section{Conclusions}

The presented brain model is easy to replicate, inexpensive, anatomically accurate, and provides a medium for neurosurgeons to teach and practice ventricular catheter placement in a risk-free environment.

\section{References}

1. Camacho EF, Boszczowski I, Freire MP, et al. Impact of an educational intervention implanted in a neurological intensive care unit on rates of infection related to external ventricular drains. PLoS One. 2013;8(2):e50708.
2. Chai FY, Farizal F, Jegan T. Coma due to malplaced external ventricular drain. Turk Neurosurg. 2013;23(4):561-563.

3. Jamjoom AAB, Joannides AJ, Poon MT-C, et al. Prospective, multicentre study of external ventricular drainage-related infections in the UK and Ireland. J Neurol Neurosurg Psychiatry. 2018;89(2):120-126.

4. Lemole GM Jr, Banerjee PP, Luciano C, et al. Virtual reality in neurosurgical education: part-task ventriculostomy simulation with dynamic visual and haptic feedback. Neurosurgery. 2007;61(1):142-149.

5. Foreman PM, Hendrix P, Griessenauer CJ, et al. External ventricular drain placement in the intensive care unit versus operating room: evaluation of complications and accuracy. Clin Neurol Neurosurg. 2015;128:94-100.

6. Schirmer CM, Elder JB, Roitberg B, Lobel DA. Virtual reality-based simulation training for ventriculostomy: an evidence-based approach. Neurosurgery. 2013;73(suppl 1):66-73

7. Tai BL, Rooney D, Stephenson F, et al. Development of a 3D-printed external ventricular drain placement simulator: technical note. J Neurosurg. 2015;123(4):1070-1076.

8. Yudkowsky R, Luciano C, Banerjee P, et al. Practice on an augmented reality/haptic simulator and library of virtual brains improves residents' ability to perform a ventriculostomy. Simul Healthc. 2013;8(1):25-31.

9. Herrick DB, Ullman N, Nekoovaght-Tak S, et al. Determinants of external ventricular drain placement and associated outcomes in patients with spontaneous intraventricular hemorrhage. Neurocrit Care. 2014;21(3):426-434.

10. Huyette DR, Turnbow BJ, Kaufman C, et al. Accuracy of the freehand pass technique for ventriculostomy catheter placement: retrospective assessment using computed tomography scans. J Neurosurg. 2008;108(1):88-91.

11. Wilson MP, O'Kelly C, Jack AS, Rempel J. Utilizing preprocedural CT scans to identify patients at risk for suboptimal external ventricular drain placement with the freehand insertion technique. J Neurosurg. 2019;130(6):2048-2054.

12. Zerbato D, Dall'Alba D. Role of virtual simulation in surgical training. J Vis Surg. 2017;3:23.

\section{Disclosures}

The authors report no conflict of interest concerning the materials or methods used in this study or the findings specified in this paper.

\section{Author Contributions}

Conception and design: Todnem, Reddy, Alleyne. Acquisition of data: Nguyen, Reddy, Grogan, Waitt. Analysis and interpretation of data: Nguyen, Reddy, Grogan, Waitt. Drafting the article: Nguyen, Todnem, Reddy, Grogan, Waitt. Critically revising the article: Nguyen. Reviewed submitted version of manuscript: Todnem, Alleyne.

\section{Correspondence}

Khoi D. Nguyen: Augusta University Medical Center, Augusta, GA.khnguyen@augusta.edu. 